\title{
Observational Proofs with Critical Contexts
}

\author{
Narjes Berregeb Adel Bouhoula Michaël Rusinowitch \\ LORIA - INRIA Lorraine \\ 615, rue du Jardin Botanique - B.P. 101 \\ 54602 Villers-lès-Nancy Cedex, France. \\ E-mail:\{berregeb,bouhoula,rusi\}@loria.fr
}

\begin{abstract}
Observability concepts contribute to a better understanding of software correctness. In order to prove observational properties, the concept of Context Induction has been developed by Hennicker [10]. We propose in this paper to embed Context Induction in the implicit induction framework of [8]. The proof system we obtain applies to conditional specifications. It allows for many rewriting techniques and for the refutation of false observational conjectures. Under reasonable assumptions our method is refutationally complete, i.e. it can refute any conjecture which is not observationally valid. Moreover this proof system is operational: it has been implemented within the Spike prover and interesting computer experiments are reported.
\end{abstract}

\section{Introduction}

Observational concepts are fundamental in formal methods since for proving the correctness of a program with respect to a specification it is essential to be able to abstract away from internal implementation details. Data objects can be viewed as equal if they cannot be distinguished by experiments with observable result. The idea that the semantics of a specification must describe the behaviour of an abstract data type as viewed by an external user, is due to [9]. Though a lot of work has been devoted to the semantical aspects of observability (see [2] for a classification), few proof techniques have been studied $[17,5,14,13]$, and even less have been implemented. In this paper we propose an automatic method for proving observational properties of conditional specifications. The method relies on computing families of well chosen contexts, called critical contexts, that "cover" in some sense all observable ones. These families are applied as induction schemes. Our inference system basically consists in extending terms by critical contexts and simplifying the results with a powerful rewriting machinery in order to generate new subgoals. An advantage of this approach is that it allows also for disproving false observational conjectures. The method is even refutationally complete for an interesting class of specifications. From a preliminary implementation on top of the Spike prover [8] computer experiments are reported. The given examples have been treated in a fully automatic way by the program. 


\section{Related works}

Hennicker [10] has proposed an induction principle, called context induction, which is a proof principle for behavioural abstractions. A property is observationally valid if it is valid for all observable experiments. Such experiments are represented by observable contexts, which are context of observable sort over the signature of a specification where a distinguished subset of its sorts is specified as observable. Hence, a property is valid for all observable experiments if it is valid for all corresponding observable contexts. A context $c$ is viewed as a particular term containing exactly one variable; therefore, the subterm ordering defines a noetherian relation on the set of observable contexts. Consequently, the principle of structural induction induces a proof principle for properties of contexts of observable sort, which is called context induction. This approach provides with a uniform proof method for the verification of behavioural properties. It has been implemented in the system ISAR [1]. However, in concrete examples, this verification is a non trivial task, and requires human guidance: the system often needs a generalization of the current induction assertion before each nested context induction, so that to achieve the proof.

Malcolm and Goguen [14] have proposed a proof technique which simplifies Hennicker proofs. The idea is to split the signature into generators and defined functions. Proving that two terms are behaviourally equivalent, comes to prove that they give the same result in all observable contexts built from defined functions, provided that the generators verify a congruence relation w.r.t. behavioural equivalence. This proof technique is an efficient optimization of Hennicker proofs.

Bidoit and Henniker [4] have investigated how a first order logic theorem prover can be used to prove properties in an observational framework. The method consists in computing automatically some special contexts called $\mathrm{cru}$ cial contexts, and in enriching the specification so that to automatically prove observational properties. But this method was only developed for the proof of equations and for specifications where only one sort is not observable. Besides, it fails on several examples (cf. Stack example), where it is not possible to compute crucial contexts.

Bidoit and Hennicker [5] have also investigated characterization of behavioural theories that allows for proving behavioural theorems with standard proof techniques for first order logic. In particular they propose general conditions under which an infinite axiomatization of the observational equality can be transformed into a finitary one. However, in general there is no automatic procedure for generating such a finite axiomatization of the observational equality.

Puel [16] has adapted Huet-Hullot procedure for proof by consistency w.r.t. the final model. Lysne [13] extends Bachmair's method for proof by consistency to the final algebra framework. The proof technique is based on a special completion procedure whose idea is to consider, not only critical pairs emerging from positioning rewrite rules on equations, but also those emerging from positioning equations on to rewrite rules. This approach is restricted to equations and requires the ground convergence property of the axioms in order to be sound (in our case, ground convergence is needed only for refutational completeness). 


\section{Basic notions}

We assume that the reader is familiar with the basic concepts of algebraic specifications [18], term rewriting and equational reasoning. A many sorted signature $\Sigma$ is a pair $(S, F)$ where $S$ is a set of sorts and $F$ is a set of function symbols. For short, a many sorted signature $\Sigma$ will simply be denoted by $F$. We assume that we have a partition of $F$ in two subsets, the first one, $C$, contains the constructor symbols and the second, $D$, is the set of defined symbols. Let $X$ be a family of sorted variables and let $T(F, X)$ be the set of sorted terms. $\operatorname{var}(t)$ stands for the set of all variables appearing in $t$. A term is linear if all its variables occur only once in it. If $\operatorname{var}(t)$ is empty then $t$ is a ground term. The set of all ground terms is $T(F)$. Let $A$ be an arbitrary non-empty set, and let $F_{A}=\left\{f_{A} \mid f \in F\right\}$ such that if $f$ is of arity $n$ then $f_{A}$ is a function from $A^{n}$ to $A$. The pair $(A, F)$ is called a $\Sigma$-algebra, and $A$ the carrier of the algebra. For sake of simplicity, we will write $A$ to denote the $\Sigma$-algebra when $F$ and $F_{A}$ are non-ambiguous.

A substitution assigns terms of appropriate sorts to variables. The domain of $\eta$ is defined by: $\operatorname{dom}(\eta)=\{x \mid x \eta \neq x\}$. If $t$ is a term, then $t \theta$ denotes the application of $\theta$ to $t$. If $\eta$ applies every variable to a ground term, then $\eta$ is a ground substitution. We denote by $\equiv$ the syntactic equivalence between objects. Let $N^{*}$ be the set of sequences of positive integers. For any term $t, \operatorname{Pos}(t) \subseteq N^{*}$ denotes its set of positions and the expression $t / u$ denotes the subterm of $t$ at a position $u$. We write $t[s]_{u}$ (resp. $t[s]$ ) to indicate that $s$ is a subterm of $t$ at position $u$ (resp. at some position).The top position is written $\varepsilon$. Let $t(u)$ denote the symbol of $t$ at position $u$. A position $u$ in a term $t$ is said to be $a$ strict position if $t(u)=f \in F$. A position $u$ in a term $t$ such that $t(u)=x$ and $x \in X$, is a linear variable position if $x$ occurs only once in $t$, otherwise, $u$ is a non linear variable position. The depth of a term $t$ is defined as follows: $|t|=0$ if $t$ is a constant or a variable, otherwise, $\left|f\left(t_{1}, \ldots, t_{n}\right)\right|=1+\max _{i}\left|t_{i}\right|$. We denote by $\succ$ a transitive irreflexive relation on the set of terms, that is noetherian, monotonic $\left(s \succ t\right.$ implies $\left.w[s]_{u} \succ w[t]_{u}\right)$, stable per instantiation $(s \succ t$ implies $s \sigma \succ t \sigma)$ and satisfies the subterm property $(f(\cdots, t, \cdots) \succ t$ ). The multiset extension of $\succ$ will be denoted by $\gg$. An equation is a formula of the form $l=r$. A conditional equation is a formula of the following form: $\bigwedge_{i=1}^{n} a_{i}=b_{i} \Rightarrow l=r$. It will be written $\bigwedge_{i=1}^{n} a_{i}=b_{i} \Rightarrow l \rightarrow r$ and called a conditional rule if $\{l \sigma\} \gg\left\{r \sigma, a_{1} \sigma, b_{1} \sigma, \cdots, a_{n} \sigma, b_{n} \sigma\right\}$ for each substitution $\sigma$. The precondition of rule $\bigwedge_{i=1}^{n} a_{i}=b_{i} \Rightarrow l \rightarrow r$ is $\bigwedge_{i=1}^{n} a_{i}=b_{i}$. The term $l$ is the left-hand side of the rule. A rewrite rule $c \Rightarrow l \rightarrow r$ is left-linear if $l$ is linear. A set of conditional rules is called a rewrite system. A constructor is free if it is not the root of a left-hand side of a rule. Let $t$ be a term in $T(C, X), t$ is called a constructor term. A rewrite system $R$ is left-linear if every rule in $R$ is left-linear. We define $\operatorname{depth}(R)$ as the maximal depth of the strict positions in its left-hand sides. Let $R$ be a set of conditional rules. Let $t$ be a term and $u$ a position in $t$. We write: $t[l \sigma]_{u} \rightarrow_{R} t[r \sigma]_{u}$ if there is a substitution $\sigma$ and a conditional equation $\bigwedge_{i=1}^{n}: a_{i}=b_{i} \Rightarrow l=r$ in $R$ such that:

(i) $l \sigma \succ r \sigma$.

(ii) for all $i \in[1 \cdots n]$ there exists $c_{i}$ such that $a_{i} \sigma \rightarrow_{R}^{*} c_{i}$ and $b_{i} \sigma \rightarrow_{R}^{*} c_{i}$. 
(iii) $\left\{t[l \sigma]_{u}\right\} \gg\left\{a_{1} \sigma, b_{1} \sigma, \cdots, a_{n} \sigma, b_{n} \sigma\right\}$.

Rewriting is extended to literals and clauses as expected.

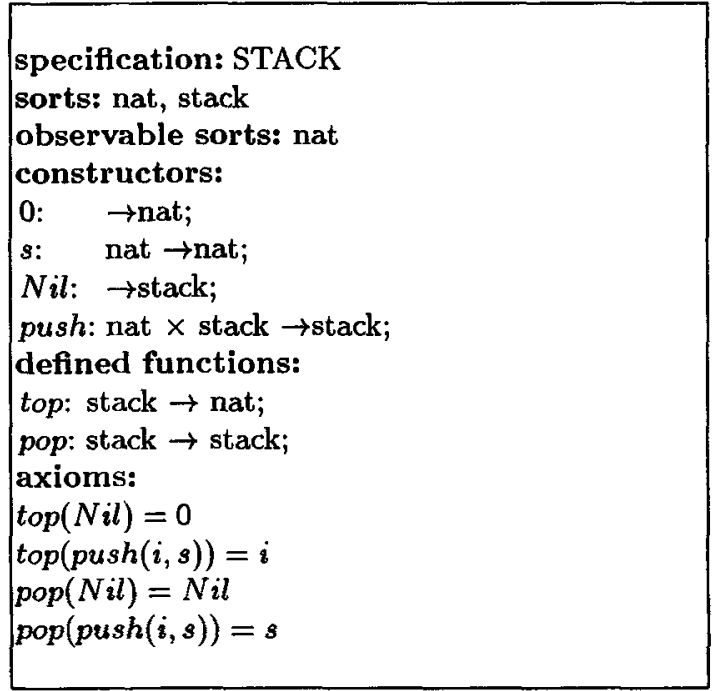

Fig. 1. Stack specification

A term $t$ is irreducible (or in normal form) if there is no term $s$ such that $t \rightarrow_{R}$ $s$. A term $t$ is ground reducible iff all its ground instances are reducible. A symbol $f \in F$ is completely defined if all ground terms with root $f$ are reducible to terms in $T(C)$. We say that $R$ is sufficiently complete if all symbols in $D$ are completely defined. A clause $C$ is an expression of the form : $\bigwedge_{i=1}^{n} a_{i}=b_{i} \Rightarrow \bigvee_{j=1}^{m} a_{j}^{\prime}=b_{j}^{\prime}$. The clause $C$ is positive if $n=0$. The clause $C$ is a logical consequence of $E$ if $C$ is valid in any model of $E$, denoted by $E \vDash C$. We say that $C$ is inductively valid in $E$ and denote it by $E \vDash_{\text {Ind }} C$ if for any ground substitution $\sigma$, (for all $i, E \vDash a_{i} \sigma=b_{i} \sigma$ ) implies (there exists $j, E \models a_{j}^{\prime} \sigma=b_{j}^{\prime} \sigma$ ). We say that two terms $s$ and $t$ are joinable, denoted by $s \downarrow_{R} t$, if $s \rightarrow_{R}^{*} v$ and $t \rightarrow_{R}^{*} v$ for some term $v$. The rewrite system $R$ is ground convergent if the terms $u$ and $v$ are joinable whenever $u, v \in T(F)$ and $R \vDash u=v$.

\section{Observational semantics}

The notion of observation technique have been introduced as a means for describing what is observed in a given algebra. An observational specification is then obtained by adding an observation technique to a standard algebraic specification. The observation technique we use in our method is based on sorts ${ }^{1}$. The semantics we choose is based on a relaxing of the satisfaction relation. The

\footnotetext{
${ }^{1}$ but it can be easily extended to observations based on operators
} 
notion of context is fundamental in all approaches based on such observational semantics. An observational property is obtained by taking into account only observable information. Thus, to show that it is valid, one has to show its validity in all observable contexts.

Let $T(F, X)$ be a term algebra and let $(S, F)$ be its signature. A context over $F$ is a non-ground term $c \in T(F, X)$ with a distinguished occurrence of a variable called the context variable of $c$. To indicate the context variable $z_{s}$ occuring in $c$, we often write $c\left[z_{s}\right]$ instead of $c$, where $s$ is the sort of $z_{s}$. A context reduced to a variable $z_{s}$ of sort $s$ is called an empty context of sort $s$. The application of a context $c\left[z_{s}\right]$ to a term $t \in T(F, X)$ of sort $s$, denoted by $c[t]$, is defined by the substitution of $z_{s}$ by $t$ in $c\left[z_{s}\right]$. The context $c$ is said to be applicable to $t$. By exception, $\operatorname{var}(c)$ will denote the set of all variables occurring in $c$ but the context variable of $c$. A context $c$ is ground if $\operatorname{var}(c)=\emptyset$. We denote by $|c|$ the depth of $c$. A subcontext (resp. strict subcontext) of $c$, is a subterm (resp. strict subterm) of $c$ with the same contextual variable. A clausal context $c$ for a clause $C$ is a list of contexts $\left\langle c_{1}, \ldots, c_{n}, c_{1}^{\prime}, \ldots, c_{m}^{\prime}>\right.$ such that for all $i \in[1 . . n]: c_{i}$ is applicable to $a_{i}=b_{i}$, and for all $j \in[1 . . m]: c_{j}^{\prime}$ is applicable to $a_{j}^{\prime}=b_{j}^{\prime}$. The application of $c$ to $C$, denoted by $c[C]$, gives the clause $\bigwedge_{i=1}^{n} c_{i}\left[a_{i}\right]=c_{i}\left[b_{i}\right] \Rightarrow \bigvee_{j=1}^{m} c_{j}^{\prime}\left[a_{i}^{\prime}\right]=c_{i}^{\prime}\left[b_{i}^{\prime}\right]$. Let $c\left[z_{s}\right]$ and $c^{\prime}\left[z_{s^{\prime}}^{\prime}\right]$ be two contexts such that $c$ is of sort $s^{\prime}$, let $t$ be a term of sort $s$ and $\sigma$ be a substitution such that $z_{s} \notin \operatorname{dom}(\sigma)$. We use the following notations: $c^{\prime}[(c[t])]=\left(c^{\prime}[c]\right)[t]=c^{\prime}[c[t]]$ and $(c[t]) \sigma=(c \sigma)[t \sigma]=c[t] \sigma$.

A specification $S P$ is a triple $(S, F, E)$ where $(S, F)$ is a signature and $E$ is a set of conditional equations. An observational specification $S P_{o b s}$ is a couple $\left(S P, S_{o b s}\right)$ such that $S P=(S, F, E)$ is a specification and $S_{o b s} \subseteq S$ is the set of observable sorts. The Stack specification in Figure 1, is an observational specification, where $S_{o b s}=\{n a t\}$.

An observable term is a term whose sort belongs to $S_{\text {obs }}$. The set of observable contexts is denoted by $C_{o b s}$. An equation $a=b$ is observable if $a$ and $b$ are observable. The precondition of a rule is observable if all its equations are observable. Consider the specification in Figure 1. There are infinitely many observable contexts: $\operatorname{top}\left(z_{\text {stack }}\right), \operatorname{top}\left(p o p\left(z_{\text {stack }}\right)\right), \ldots$, top $\left(p o p\left(\ldots\left(p o p\left(z_{\text {stack }}\right)\right) \ldots\right)\right), \ldots \operatorname{top}\left(p u s h\left(z_{\text {stack }}\right)\right), \operatorname{top}\left(p u s h\left(i, p o p\left(z_{\text {stack }}\right)\right)\right), \ldots$

The notion of observational validity is based on the idea that two objects in a given algebra are observationally equal if they cannot be distinguished by computations with observable results. These computations are formalized by contexts.

Let $a$ and $b$ be two terms. We say that $a$ and $b$ are observationally equal, and we denote it by $E \vDash F_{b s} a=b$ iff for all $c \in C_{o b s}, E \vDash$ Ind $c[a]=c[b]$. Consider the stack specification in Figure 1. It is easy to see that $p u s h(\operatorname{top}(s), \operatorname{pop}(s))=s$ is not satisfied (in the classical sense). However, intuitively, it is observationally satisfied if we just observe the elements of the sequences $p u s h(t o p(s), \operatorname{pop}(s))$ and $s$. This can be formally shown by considering all observable contexts.

The next theorem gives a useful characterization of observational theorems (see e.g. [15]): 
Theorem 1. Suppose that all the preconditions of $E$ are observable. Then $E \vDash$ obs $\bigwedge_{i=1}^{n} a_{i}=b_{i} \Rightarrow \bigvee_{j=1}^{m} a_{j}^{\prime}=b_{j}^{\prime}$ iff for all ground substitutions $\sigma$, if (for all $i, E \vDash \models_{O b s} a_{i} \sigma=b_{i} \sigma$ ) then (there exists $j$ such that $E \vDash o b s a_{j}^{\prime} \sigma=$ $\left.b_{j}^{\prime} \sigma\right)$.

\section{Induction schemes}

Our purpose in this section is to introduce the ingredients allowing us to prove and disprove behavioural properties. This task amounts in general to check an infinite number of ground formulas for validity, since an infinite number of instances and an infinite number of contexts have to be considered for building these ground instances. This is where induction comes into play. Test substitutions will provide us with induction schemes for substitutions and critical contexts will provide us with induction schemes for contexts. In general, it is not possible to consider all the observable contexts. However, cover contexts are sufficient to prove behavioural theorems by reasoning on the ground irreducible observable contexts rather than on the whole set of observable contexts. In the following, we denote by $R$ a conditional rewriting system with observable preconditions.

Definition 2 (cover set). A cover set, denoted by $C S$, for $R$, is a finite set of irreducible terms such that for all ground irreducible term $s$, there exist a term $t$ in $C S$ and a ground substitution $\sigma$ such that $t \sigma \equiv s$.

We now introduce the notion of cover context that is used to schematize all contexts. Note that a cover context need not be observable, (unlike crucial contexts of [4]). The intuitive idea is to use cover context to extend the conjectures by the top in order to create redexes. Then the obtained formulas can be simplified by axioms and induction hypothesis.

Definition 3. [cover context set] A cover context set $C C$ is a set of contexts such that: for each ground irreducible context $c_{o b s}\left[z_{s}\right] \in C_{o b s}$, there exists $c\left[z_{s}\right] \in C C$ and a substitution $\theta$ such that $\operatorname{dom}(\theta)=\operatorname{var}(c)$ and $c \theta$ is a subcontext of $c_{o b s}$.

A cover context set for the specification stack is $\left\{z_{\text {nat }}, \operatorname{top}\left(z_{s t a c k}\right), \operatorname{pop}\left(z_{s t a c k}\right)\right\}$. The context $p u s h\left(i, z_{s t a c k}\right)$ is not a cover context since top $\left(p u s h\left(i, z_{s t a c k}\right)\right)$ and $\operatorname{pop}\left(p u s h\left(i, z_{s t a c k}\right)\right)$ are reducible. Note that usually there are infinitely many possible cover context sets. For instance, $\left\{z_{\text {nat }}, \operatorname{top}\left(z_{s t a c k}\right), \operatorname{top}\left(\operatorname{pop}\left(z_{\text {stack }}\right)\right), \operatorname{pop}\left(\operatorname{pop}\left(z_{\text {stack }}\right)\right)\right\}$ is also a cover context set.

In the following, we refine cover context sets so that to be able not only to prove behavioural properties, but also to disprove the non valid ones. We need first to introduce the following notions: A context $c$ is quasi ground reducible if for all ground substitution $\tau$ such that $\operatorname{dom}(\tau)=\operatorname{var}(c), c \tau$ is reducible. A term $t$ is strongly irreducible if none of its non-variable subterms matches a left-hand side of a rule in $R$. A positive clause $C_{p o s} \equiv \mathrm{V}_{i=1}^{n} a_{i}=b_{i}$ is strongly irreducible 
if $C_{p o s}$ is not a tautology, and the maximal elements of $\left\{a_{i}, b_{i}\right\}$ w.r.t. $\prec$ are strongly irreducible by $R$.

Cover sets and cover context sets are fundamental for the correctness of our method. However, they cannot help us to disprove the non observationally valid clauses. For this purpose, we introduce a new notion of critical context sets and we use test sets defined in [7].

Definition 4 (test set, test substitution). A test set is a cover set which has the following additional properties: (i) the instance of a ground reducible term by a test substitution matches a left-hand side of $R$. (ii) if the instance of a positive clause $C_{\text {pos }}$ by a test substitution $\sigma$ is strongly irreducible, then $C_{p o s} \sigma$ is not inductively valid w.r.t. $R$. A test substitution for a clause $C$ instanciates all induction variables of $C$ by terms taken from a given test set whose variables are renamed.

Definition 5 (critical context set, critical clausal context). A critical context set $S$ is a cover context set such that for each positive clause $C_{\text {pos }}$, if $c\left[C_{p o s}\right] \sigma$ is strongly irreducible where $\sigma$ is a test substitution of $C_{p o s}$ and $c$ is a clausal context of $C_{\text {pos }}$, then $C_{p o s} \sigma$ is not observationally valid w.r.t. $R$. A critical clausal context for a clause $C$ is a clausal context for $C$ whose contexts belongs to $S$.

Test substitutions and critical context sets permit us to refute false conjectures by constructing a counterexample.

Definition 6 (provably inconsistent). Let $R$ be a conditional rewriting system with observable preconditions. We say that $C \equiv \bigwedge_{i=1}^{n} a_{i}=b_{i} \Rightarrow \bigvee_{j=1}^{m} a_{j}^{\prime}=$ $b_{j}^{\prime}$ is provably inconsistent if and only if there exists a test substitution $\sigma$ and a clausal critical context $c$ such that:

(i) for all $i, a_{i} \sigma=b_{i} \sigma$ is an inductive theorem w.r.t. $R$.

(ii) $c\left[\mathrm{~V}_{j=1}^{m} a_{j}^{\prime}=b_{j}^{\prime}\right] \sigma$ is strongly irreducible by $R$.

Provably inconsistent clauses are not observationally valid.

Theorem 7. Let $R$ be a conditional rewriting system with observable preconditions. Let $C$ be a provably inconsistent clause. Then $C$ is not observationally valid.

\subsection{Computation of test sets}

The computation of test sets and test substitutions for conditional specifications is decidable if the axioms are sufficiently complete and the constructors are specified by a set of unconditional equations (see [12]). Unfortunately, no algorithm exists for the general case of conditional specifications. However, in [7], a procedure is described for computing test sets when the axioms are sufficiently complete over an arbitrary specification of constructors. 


\subsection{Computation of critical contexts}

Let us first introduce the following lemma which gives us a useful characterization of critical context sets:

Lemma 8. Let $R$ be a conditional rewriting system. Let $C C$ be a cover context set that has the following properties:

(i) any non-observable context in CC has variables at depth greater than or equal to depth $(R)$.

(ii) for each context $c\left[z_{s}\right] \in C C$, there exists an observable context $c_{o b s}$ such that $c_{o b s}[c]$ is strongly irreducible.

Then, $C C$ is a critical context set for $R$.

Proof. Let $C$ be a positive clause such that $c[C] \sigma$ is strongly irreducible, where $\sigma$ is a test substitution of $C$ and $c$ is a critical clausal context of $C$. Let us prove that $C \sigma$ is not observationally valid. By (ii), there exists an observable clausal context $c_{o b s}$ such that $c_{o b s}[c]$ is strongly irreducible. Now, using (i), we conclude that $c_{o b s}[c[C]] \sigma$ is also strongly irreducible. Then, $c_{o b s}[c[C]] \sigma$ is a provably inconsistent clause w.r.t. Definition 11 in [7]. By Theorem 12 in [7], $c_{o b s}[c[C]] \sigma$ is not inductively valid. Thus, $R \nvdash_{O b s} C \sigma$.

$C C_{0}:=\left\{c \in T(F, X) \quad|\quad| c \mid \leq \operatorname{depth}(R), \quad c \in C_{o b s}, \quad c\right.$ is not quasi ground reducible, and does not contain any observable strict subcontext $\}$

$T_{0}:=\left\{c \in T(F, X) \quad|\quad| c \mid=\operatorname{depth}(R), \quad c \notin C_{o b s}, \quad c\right.$ is not quasi ground reducible, $c$ does not contain any observable subcontext, and all variables (including the context one) in c occur at depth $(R)\}$.

repeat

$C C_{i+1}:=C C_{i} \cup\left\{c \in T_{i} \mid \exists c_{i} \in C C_{i}\right.$ such that $c_{i}[c]$ is not quasi ground reducible $\}$

$T_{i+1}:=T_{i} \backslash C C_{i+1}$

until $C C_{i+1}=C C_{i}$

output: $C C_{i}$

Fig. 2. Computation of Critical Contexts

Now, let us present our method for constructing such critical contexts. The idea of our procedure is the following: starting from the non quasi ground reducible observable contexts of depth smaller than or equal to $\operatorname{depth}(R)$, we construct all contexts that can be embedded in one of those observable contexts, to give a non quasi ground reducible and observable context. It can be proved, by 
reduction to ground reducibility, that quasi ground reducibility is decidable too for equational systems and semi-decidable for conditional rewrite systems [11]. The following remark is also useful: given a context $c\left[z_{s}\right]$ of the form $f\left(t_{1}, \ldots, t_{n}\right)$ where $f$ is a completely defined function and for all $i \in[1 . . n], t_{i}$ is a constructor tęrm. If $z_{s}$ does not appear at an induction position of $f$, then $c\left[z_{s}\right]$ is quasi ground reducible.

Theorem 9. Let $R$ be a rewriting system and $C C$ be the result of the application of the procedure given in Figure 2. Then:

- $C C$ is a cover context set for $R$.

- if $R$ is equational and left-linear then $C C$ is a critical context set for $R$.

Proof. It is relatively easy to show that $C C$ is a cover context set for $R$. Now, assume that $R$ is equational and leftlinear and let us prove that $C C$ is also a critical context set for $R$. By construction, any non-observable context in $C C$ has variables at depth greater than or equal to $\operatorname{depth}(R)$. Now, since $R$ is equational, any non quasi ground reducible context is necessarily strongly irreducible. On the other hand, $R$ is left-linear and the variables of non-observable context occur at $\operatorname{depth}(R)$, then for each context $c\left[z_{s}\right] \in C C$, there exists $i$ such that $c \in C C_{i}$, we can show that there exists an observable context $c_{o b s}$ such that $c_{o b s}[c]$ is strongly irreducible. The proof is done by induction on $i$.

Example 10. Consider the Stack specification in Figure 1. We have $\operatorname{depth}(R)=$ 1, then:

$C C_{0}=\left\{z_{\text {nat }}, \operatorname{top}\left(z_{\text {stack }}\right)\right\}$

$T_{0}=\left\{p o p\left(z_{\text {stack }}\right), p u s h\left(i, z_{\text {stack }}\right)\right\}$.

$C C_{1}=\left\{z_{\text {nat }}, \operatorname{top}\left(z_{\text {stack }}\right)\right\} \cup\left\{p o p\left(z_{\text {stack }}\right)\right\}$,

$C C=C C_{1}$ is a critical context set for $R$.

Example 11. Consider the List specification in Figure 3. We have: $\operatorname{depth}(R)=1$, then:

$C C_{0}=\left\{z_{\text {nat }}, z_{\text {bool }}, i n\left(x, z_{\text {list }}\right)\right\}$,

$T_{0}=\left\{\right.$ union $\left(z_{\text {list }}, x\right)$, insert $\left.\left(x, z_{\text {list }}\right)\right\}$,

$C C_{1}=C C_{0} \cup\left\{\right.$ union $\left.\left(z_{\text {list }}, x\right)\right\}$.

$C C=C C_{1}$ is a cover context set for $R$. In fact, $\operatorname{union}\left(x, z_{\text {list }}\right)$ is quasi ground reducible and $i n\left(y\right.$, union $\left.\left(z_{l i s t}, x\right)\right)$ is not quasi ground reducible since $\operatorname{in}\left(0\right.$, union $\left.\left(z_{\text {list }}, N i l\right)\right)$ is irreducible, but $\operatorname{in}\left(y, \operatorname{insert}\left(x, z_{\text {list }}\right)\right)$ is quasi ground reducible.

It is possible to compute critical context sets in the case where $R$ is a conditional rewriting system. It is sufficient to apply our procedure given in Figure 2 to compute a cover context set $C C$, and then to check that for each non observable context $c \in C C$, there exists an observable context $c_{o b s}$ such that $c_{o b s}[c]$ is strongly irreducible. In Example 11, we have $\operatorname{in}\left(x,\left(u n i o n\left(z_{l i s t}, y\right)\right.\right.$ is strongly irreducible, then we conclude that $C C=\left\{z_{\text {nat }}, z_{\text {bool }}, \operatorname{in}\left(x, z_{\text {list }}\right)\right.$, union $\left.\left(z_{\text {list }}, x\right)\right\}$ is a critical context set for $R$. 


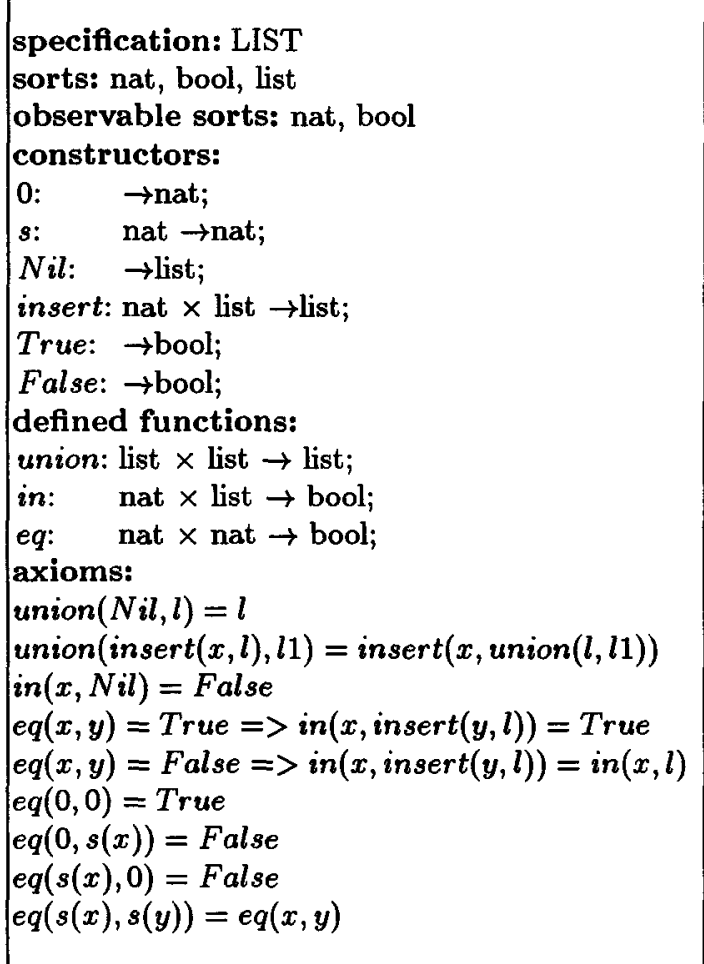

Fig. 3. List specification

\section{Inference system}

The inference system we use (see Figure 4) is based on a set of transition rules applied to $(E, H)$, where $E$ is the set of conjectures to prove and $H$ is the set of induction hypotheses. The initial set of conditional rules $R$ is oriented with a well founded ordering. An I-derivation is a sequence of states: $\left(E_{0}, \emptyset\right) \vdash_{I}\left(E_{1}, H_{1}\right) \vdash_{I}$ $\ldots\left(E_{n}, H_{n}\right) \vdash_{I} \ldots$. We say that an I-derivation is fair if the set of persistent clauses $\left(\cup_{i} \cap_{j \geq i} E_{j}\right)$ is empty. Context induction is performed implicitly by the Generation rule. An equation is selected in a clause and it is extended by critical contexts and test sets. These extensions are rewritten by $R$ either by conditional rewriting or by case analysis. The resulting conjectures are collected in $\bigcup_{c, \sigma} E_{c, \sigma}$. Case Simplification illustrates the case reasoning: it simplifies a conjecture with conditional rules provided that the disjunction of their preconditions ${ }^{2}$ is inductively valid in $R$.

Definition 12 (Case Analysis). Let $R$ be a set of conditional rules and let $l \vee r$ be a clause. Case Analysis $\left(l[g \sigma]_{u}, r\right)=\left\{P_{1} \sigma \Rightarrow l\left[d_{1} \sigma\right]_{u} \vee r ; \cdots ; P_{n} \sigma \Rightarrow\right.$ $\left.l\left[d_{n} \sigma\right]_{u} \vee r\right\}$ if $\forall i \in[1 \cdots n]: P_{i} \Rightarrow g \rightarrow d_{i} \in R$ and $R \vDash F_{n d} P_{1} \sigma \vee \cdots \vee P_{n} \sigma$.

\footnotetext{
${ }^{2}$ Recall that the preconditions of the axioms in $R$ are assumed to be observable
} 
The rule Context subsumption appeared to be very useful for manipulating non orientable conjectures.

An I-derivation fails when there exists a conjecture such that no rule can be applied to it. An I-derivation succeeds if all conjectures are proved.

Theorem 13 (correctness of successful I-derivations). Let $\left(E_{0}, \emptyset\right) \vdash_{I}$ $\left(E_{1}, H_{1}\right) \vdash_{I} \ldots$ be a fair I-derivation. If it succeeds then $R \models$ obs $E_{0}$.

Proof. The proof is done by contradiction. Suppose $R \not{ }_{O b s} E_{0}$ and let $C \in \cup_{i} E_{i}$ be a minimal counterexample w.r.t. a well founded ordering on clauses extending $\succ$ p. We can easily show, as in [8], that no inference rule can be applied to $C$. Hence $C$ persists in the derivation contradicting the fairness hypothesis.

Theorem 14 (correctness of disproof). Let $\left(E_{0}, \emptyset\right) \vdash_{I}\left(E_{1}, H_{1}\right) \vdash_{I} \cdots$ be an I-derivation. If there exists $j$ such that Disproof is applied to $\left(E_{j}, H_{j}\right)$, then $R \forall$ Obs $E_{0}$.

Proof. If there exists $j$ such that Disproof is applied to $\left(E_{j}, H_{j}\right)$, then by Theorem 7 , we conclude that $R \not \nvdash_{O b s} E_{j}$. Now, to prove that $R \not \forall_{\text {obs }} E_{0}$, it is sufficient to prove the following claim: Let $\left(E_{j}, H_{j}\right) \vdash_{I}\left(E_{j+1}, H_{j+1}\right)$ be an I-derivation step. If $\forall i \leq j, R \models$ obs $E_{i}$ then $R \models{ }_{o b s} E_{j+1}$. If $\left(E_{j}, H_{j}\right) \vdash_{I}$ $\left(E_{j+1}, H_{j+1}\right)$ by a simplification rule, then the equations which are used for simplification occur in some $E_{k}(k \leq j)$ and therefore are observationally valid in $R$ by assumption. Hence, $E_{j+1}$ is observationally valid too in $R$. If $\left(E_{j}, H_{j}\right) \vdash_{I}\left(E_{j+1}, H_{j+1}\right)$ by Generation on $C \in E_{j}$, every auxiliary equation which is used for rewriting an instance of $C$ by a critical context $c$ and a test substitution $\sigma$, is either in $R$ or $E_{k}(k \leq j)$ and hence $E_{j+1}$ is valid in $R$.

Now we consider boolean specifications. To be more specific, we assume there exists an observable sort bool with two free constructors $\{$ true, false $\}$. The sort bool will be observable. Every rule in $R$ is of type: $\bigwedge_{i=1}^{n} p_{i}=p_{i}^{\prime} \Rightarrow s \rightarrow t$ where for all $i$ in $[1 \cdots n], p_{i}^{\prime} \in\{$ true, false $\}$. Conjectures will be boolean clauses, i.e. clauses whose negative literals are of type $\neg\left(p=p^{\prime}\right)$ where $p^{\prime} \in\{$ true, false $\}$. Let $f \in D$, a completely defined symbol in $R$. Then $f$ is strongly complete [7] $w . r . t R$ if for all the rules $p_{i} \Rightarrow f\left(t_{1}, \ldots, t_{n}\right) \rightarrow r_{i}$ whose left-hand sides are identical up to a renaming $\mu_{i}$, we have $R \models_{I n d} \bigvee_{i=1}^{n} p_{i} \mu_{i}$. We say that $R$ is strongly complete if for all $f \in D, f$ is strongly complete w.r.t $R$.

Theorem 15 (refutational completeness). Let $R$ be a conditional rewrite system. Assume that $R$ is ground convergent and strongly complete. Let $E_{0}$ be $a$ set of boolean clauses. Then $R \not \nvdash_{\text {Obs }} E_{0}$ iff all fair derivations issued from $\left(E_{0}, \emptyset\right)$ fail.

Proof. The proof follows the line of the corresponding Theorem 6.5 in [6] that was given for the initial semantics. 
Generation: $\frac{(E \cup\{l \vee r\}, H)}{\left(E \cup\left(\bigcup_{c, \sigma} E_{c, \sigma}\right), H \cup\{C\}\right)}$
if $\left\{\begin{array}{l}\text { for all test substitution } \sigma \text { and for all critical context } c \text { : } \\ \text { either } c[l] \sigma \vee r \sigma \text { is a tautology, then } E_{c, \sigma}=\emptyset \\ \text { or } c[l] \sigma \rightarrow_{R} l^{\prime} \text {, then } E_{c, \sigma}=\left\{l^{\prime} \vee r \sigma\right\} \\ \text { otherwise } E_{c, \sigma}=\text { Case Analysis }(c[l] \sigma, r \sigma)\end{array}\right.$

Case Simplification: $\frac{(E \cup\{l \vee r\}, H)}{\left(E \cup E^{\prime}, H\right)}$

if $E^{\prime}=$ Case Analysis $(l, r)$

Simplification: $\frac{(E \cup\{(a=b) \vee r\}, H)}{\left(E \cup\left\{\left(a^{\prime}=b\right) \vee r\right\}, H\right)}$

if $\left\{\begin{array}{l}a \rightarrow_{R} a^{\prime} \text {, or } \\ a[v \lambda] \rightarrow_{H \cup E} a[w \lambda] \text { by } v=w \text { where } v \succ w \text { and }(v \lambda \prec a \text { or } w \lambda \prec b)\end{array}\right.$

Subsumption: $\frac{(E \cup\{C\}, H)}{(E, H)}$

if $C$ is subsumed by a clause of $R \cup H \cup E$

Context Subsumption: $\frac{(E \cup\{C\}, H)}{(E, H)}$

if $\left\{\begin{array}{l}\text { there exists a clause } C^{\prime} \in R \cup H \cup E \text { and a clausal context } c \\ \text { such that } c\left[C^{\prime}\right] \text { subsumes } C\end{array}\right.$

Delete: $\frac{(E \cup\{C\}, H)}{(E, H)}$

if $C$ is a tautology

Disproof: $\frac{(E \cup\{C\}, H)}{\text { Disproof }}$

if $C$ is provably inconsistent

Fig. 4. Inference System $I$

\section{Computer experiments}

We have implemented these results in the Spike prover, written in Caml Light.

Example 16 (Stacks). We proved automatically that push(top $(S), \operatorname{pop}(S))=S$ is a behavioural property of the stack specification (see Figure 1). Note that this example fails with the approach of [4], since it is not possible to compute automatically a set of crucial contexts: if two stacks have the same top they are not necessarily equal. In the approach of [10], we have to introduce an auxiliary function iterated_pop : nat $\times$ stack $\rightarrow$ stack such that iterated_pop $(n, s)$ iterates $n$ times pop. This is easy because pop is unary. The function iterated_pop is defined by: 
iterated_pop $(0, s)=s$, iterated_pop $(n+1, s)=$ iterated_pop $(n, p o p(s))$

Then, we have to prove the property for all contexts of the form top(iterated_pop $\left.\left(x, c\left[z_{s t a c k}\right]\right)\right)$. However, this schematization of contexts could be more complicated in case of a function of arity greater than two. So, this process seems to be not easy to automatize in general. In the approach of [14], this problem remains too.

Now, let us describe our proof. The prover computes first a test set for $R$ and the induction positions of functions, which are necessary for inductive proofs. It also computes a critical context. These computation are done only once and before the beginning of the proof.

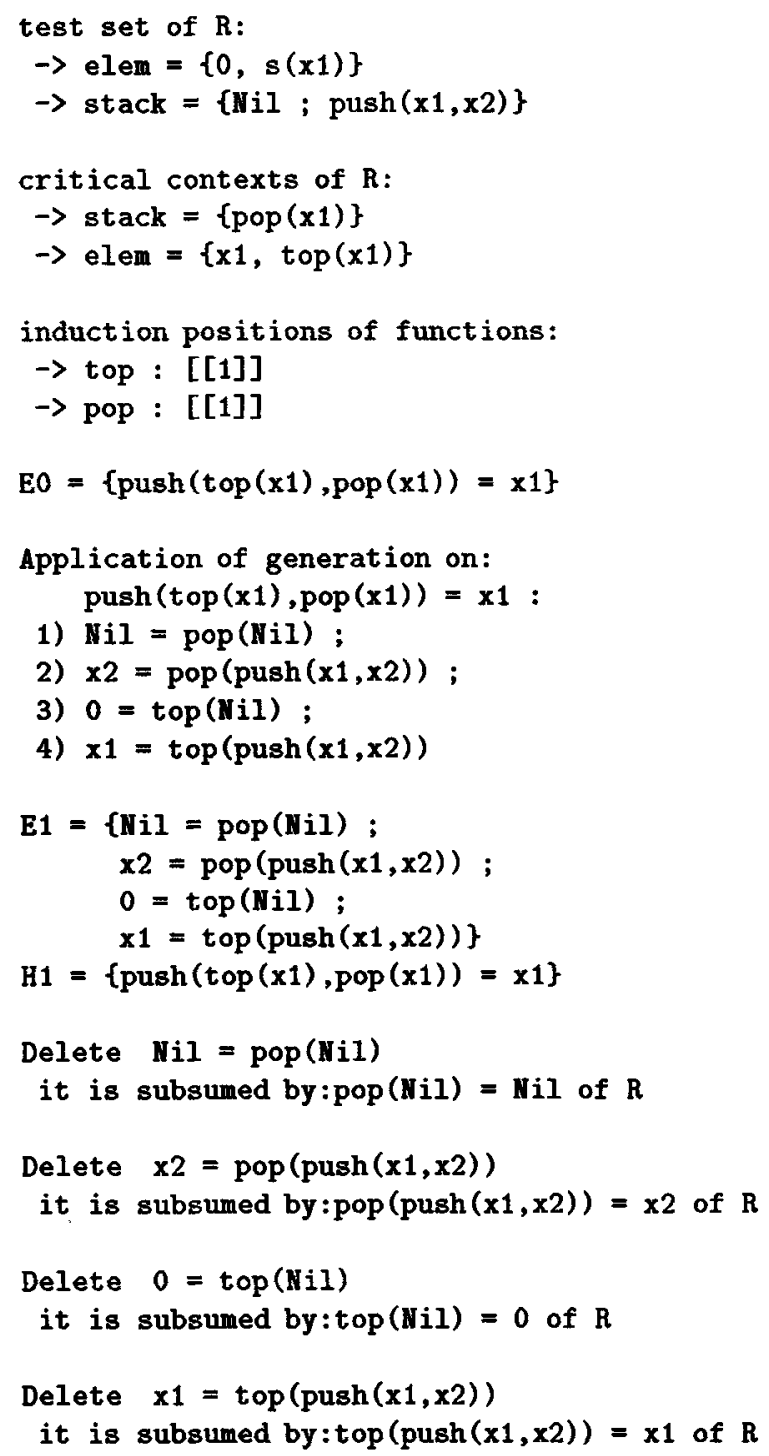


$\mathrm{E} 2=\{\}$

$H 2=\{\operatorname{push}(\operatorname{top}(x 1), \operatorname{pop}(x 1))=x 1\}$

The initial conjectures are observationally valid in $R$

Example 17 (Lists). Consider now the specification list in Figure 3. The theorem insert $(x 1, i n \operatorname{sert}(x 1, x 2))=i n \operatorname{sert}(x 1, x 2)$ is automatically proved.

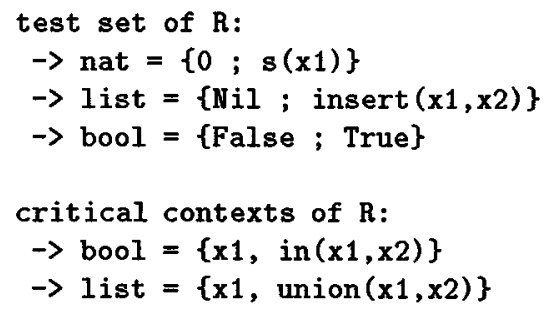

Simplification of :

insert $(x 1$, union $(x 2, x 4))=$ union(insert $(x 1, x 2), x 4)$ by $R$ :

$\operatorname{insert}(x 1, \operatorname{union}(x 2, x 4))=\operatorname{insert}(x 1, \operatorname{union}(x 2, x 4))$ 


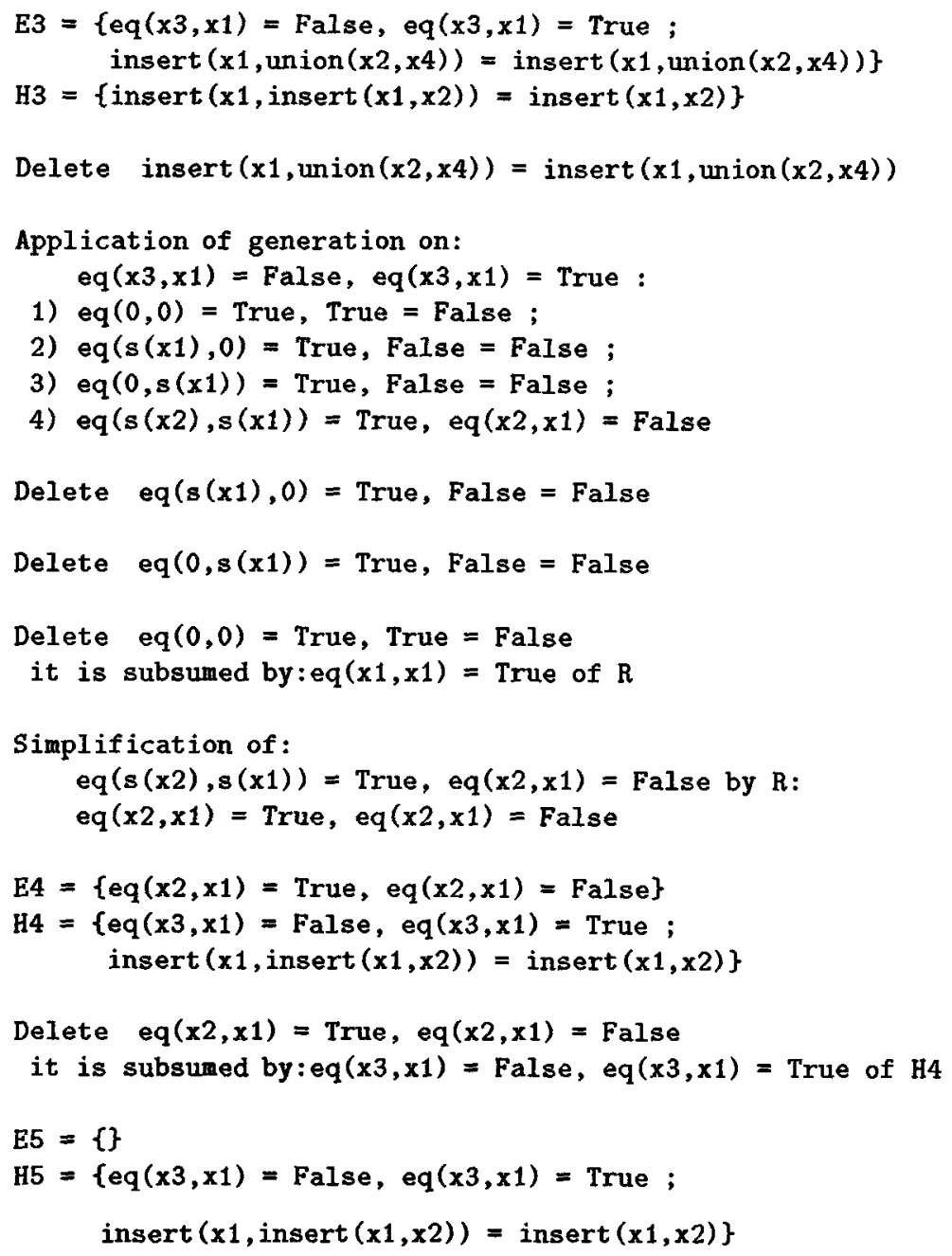

The initial conjectures are observationally valid in $R$

In the same way we have proved the following conjectures:

$$
\operatorname{insert}(x, \operatorname{insert}(y, l))=\operatorname{insert}(y, \operatorname{insert}(x, l)) \text { and } \operatorname{union}\left(l, l^{\prime}\right)=\operatorname{union}\left(l^{\prime}, l\right)
$$

\section{Conclusion}

We have presented an automatic procedure for proving observational properties in conditional specifications. The method relies on the construction of a set of critical contexts which enables to prove or disprove conjectures. Under reasonable hypotheses, we have shown that the procedure is refutational complete: each non observationally valid conjecture will be detected after a finite time. 
A cover context w.r.t. our definition 3 garantees the soundness of our procedure. However, cover contexts computed by our procedure may contain unecessary contexts, as in Example 17 where union $\left(z_{\text {list }}, x\right)$ is useless for observations. We plan to refine our notion of cover and critical contexts in order to select only the needed contexts.

We also plan to extend the observation technique to terms and formulas.

\section{References}

1. B. Bauer and R. Hennicker. Proving the correctness of algebraic implementations by the ISAR system. In DISCO'93, volume 722 of Lecture Notes in Computer Science, pages 2-16. Springer-Verlag, 1993.

2. G. Bernot, M. Bidoit, and T. Knapik. Behavioural approaches to algebraic specifications: A comparative study. Acta Informatica, 31(7):651-671, 1994.

3. N. Berregeb, A. Bouhoula, and M. Rusinowitch. Observational proofs by implicit context induction. Technical Report 3151, INRIA, 1997.

4. M. Bidoit and R. Hennicker. How to prove observational theorems with LP. In U. Martin and J. Wing, editors, Proc. of First International Workshop on Larch. Springer-Verlag, 1992.

5. M. Bidoit and R. Hennicker. Behavioural theories and the proof of behavioural properties. Theoretical Computer Science, 165(1):3-55, 1996.

6. A. Bouhoula. Using Induction and Rewriting to Verify and Complete Parameterized Specifications. Theoretical Computer Science, 170(1-2):245-276, 1996.

7. A. Bouhoula. Automated theorem proving by test set induction. Journal of Symbolic Computation, 23(1):47-77, 1997.

8. A. Bouhoula and M. Rusinowitch. Implicit induction in conditional theories. Journal of Automated Reasoning, 14(2):189-235, 1995.

9. J. Guttag. The specification and Application to Programming of Abstract Data Types. PhD Thesis, University of Toronto, 1975.

10. R. Hennicker. Context induction: a proof principle for behavioural abstractions and algebraic implementations. Formal Aspects of Computing, 3(4):326-345, 1991.

11. S. Kaplan and M. Choquer. On the decidability of quasi-reducibility. Bulletin of European Association for Theoretical Computer Science, 28:32-34, February 1986.

12. E. Kounalis. Testing for the ground (co-)reducibility property in term-rewriting systems. Theoretical Computer Science, 106:87-117, 1992.

13. O: Lysne. Extending Bachmair's method for proof by consistency to the final algebra. Information Processing Letters, 51:303-310, 1994.

14. G. Malcolm and J. Goguen. Proving correctness of refinement and implementation. Technical Monograph PRG-114, Oxford University Computing Laboratory, November 1994.

15. P. Padawitz. Computing in Horn Clause Theories. Springer-Verlag, 1988.

16. L. Puel. Proofs in the final algebra. IXth Colloquium on Trees in Algebra and Programming. Bordeaux, France, March 1984.

17. D.T. Sanella and A. Tarlecki. Towards formal development of ml programs: foundations and methodology. In J. Diaz and F. Orejas, editors, TAPSOF T'89, volume 352 of Lecture Notes in Computer Science, pages 375-389. Springer-Verlag, 1989.

18. M. Wirsing. Algebraic specifications. In J. van Leeuwen, A. Meyer, M. Nivat, M. Paterson, and D. Perrin, editors, Handbook of Theoretical Computer Science, volume B, chapter 13. Elsevier Science Publishers B. V. (North-Holland) and The MIT press, 1990. 